\title{
A Numerical Scheme to Compute the Divergence of the Wind Field
}

\author{
By Yuk-kwan Chan \\ Royal Observatory Hong Kong \\ (Manuscript received 29 October 1986, in revised form 12 February 1987)
}

\begin{abstract}
A new finite difference scheme is constructed for the divergence term with a view to decreasing the errors in its computation.

A divergence scheme is designed such that it will give zero divergence for any nondivergent wind field. In other words, the computational error in applying this scheme to any non-divergent wind field is zero. Since any wind field can be separated into a nondivergent part and a divergent part, the computational error in applying this scheme to any wind field is equal to the error involved with the divergent part only. At the same time, the divergent part wind is an order of magnitude smaller than the non-divergent part. Hence the error is reduced.

The new scheme is simple, especially in latitude-longitude or Mercator map projections.

The scheme has been applied to a 3-level limited area primitive equation model for Hong Kong and is found to give much improvement over the usual divergence schemes.
\end{abstract}

\section{Introduction}

It is a well known fact that the divergence term is usually the difference of two terms of an order of magnitude larger. Thus large error can result in the computation of the finite difference approximation of the divergence. If ' $\nabla$ ' stands for the finite difference analogue of the continuous operator 'Div', then, a simple representation such as

$$
\nabla \underline{v}=n m\left\{{\overline{\delta_{x}\left(\frac{u}{n}\right)}}^{y}+{\overline{\delta_{y}\left(\frac{v}{m}\right)}}^{x}\right\}
$$

is not satisfactory. Here, ' $m$ ', ' $n$ ' are map factors (Section 1-8, Haltiner and Williams, $1980)$ in the $x$ - and $y$-directions respectively and the operators ' $\delta$ ' and ' - ', are defined as follows:

$$
\begin{aligned}
& \delta_{x} u_{i j}=\frac{u_{i+1 / 2, j}-u_{i-1 / 2, j}}{x}, \\
& \delta_{y} v_{i j}=\frac{v_{i, j+1 / 2}-v_{i, j-1 / 2}}{y},
\end{aligned}
$$

$$
\begin{aligned}
& {\overline{u_{i j}}}^{x}=\frac{u_{i+1 / 2, j}+u_{i-1 / 2, j}}{2}, \\
& {\overline{u_{i j}}}^{y}=\frac{u_{i, j+1 / 2}+u_{i, j-1 / 2}}{2} .
\end{aligned}
$$

If (1.1) is used to compute the divergence of a geostrophic wind field, the values of the divergence obtained is comparable in order of magnitude to those of an ordinary wind field even though the former should be zero.

To decrease the error in the computation of the divergence, Y.K. Chan (1984) proposed a finite difference scheme ' $\nabla_{c}$ ' which gave zero divergence for any non-divergent wind field. In other words, this scheme is exact for any non-divergent wind field. The philosophy that this scheme will decrease the computational error for other divergent wind field is briefly outlined as follows. Since any wind field can be decomposed into the sum of the rotational component $\underline{v}_{\psi}$ and the divergent component $\underline{v} x$, the application of $\nabla_{C}$ to $\underline{v}$ gives 


$$
\nabla_{c} \underline{v}=\nabla_{c}\left(\underline{v}_{\psi}+\underline{v}_{\chi}\right)=\operatorname{Div} \underline{v}_{\chi}+\varepsilon_{\chi}
$$

where $\nabla_{c} \underline{v}$ equals zero and $\varepsilon_{x}$ is the error associated with the divergent component. If another finite difference divergence scheme $\nabla^{\prime}$ is applied to the same wind field $\underline{v}$,

$$
\nabla^{\prime} \underline{v}=\varepsilon_{\psi}^{\prime}+\operatorname{Div} \underline{v}_{\chi}+\varepsilon_{\chi}^{\prime}
$$

where $\varepsilon_{x}^{\prime}$ and $\varepsilon_{\psi}^{\prime}$ are the errors associated with the divergent and the rotational components respectively. In general, $\underline{v}_{x}$ is an order of magnitude smaller than $\underline{v}_{\psi}$ and there is no reason to assume that $\varepsilon_{\chi}$ and $\varepsilon_{\chi}^{\prime}$ differ much in order of magnitude. Thus, $\varepsilon_{\chi}$ is smaller than $\varepsilon_{x}^{\prime}+\varepsilon_{\psi}^{\prime}$. In other words, $\nabla_{c}$ approximates the divergence with smaller error than any other schemes.

However, Chan derived the finite difference scheme for the latitude-longitude projection only. This paper attempts to generalize the scheme for any map projection.

\section{Formulation}

The principle of the derivation of the scheme $\nabla$ to give zero divergence for nondivergent wind fields is simple. It is outlined as follows. In general, $\nabla \underline{v}$ takes the form of $\sum_{i, j} \alpha_{i j} v_{i j}$ which approaches Div $\underline{v}$ in the limit when the grid dimensions $\Delta x, \Delta y$ approach zero:

$$
\nabla \underline{v}=\sum_{i, j} \alpha_{i j} v_{i j} .
$$

If $\alpha_{i j}$ can be determined such that (2.1) vanishes for any non-divergent wind field $\underline{v}$, then (2.1) is the required expression.

For any non-divergent wind field $\underline{v}$, there exist a scalar field $\psi$ such that

$$
\underline{v}=\underline{k} \times \underline{\nabla} \psi \text {. }
$$

Substituting (2.2) into (2.1), $\nabla \underline{v}$ becomes an expression in $\psi_{k l}$ with coefficients $C_{k l}\left(\alpha_{i j}\right)$ which are expressions in $\alpha_{i j}$ :

$$
\nabla \underline{v}=\sum_{k, l} C_{k l}\left(\alpha_{i j}\right) \phi_{k l} .
$$

For (2.3) to vanish for arbitrary $\psi_{k l}, C_{k l}\left(\alpha_{i j}\right)$ must vanish. Thus, $\alpha_{i j}$ can be determined by solving the following set of simultaneous equations :

$$
C_{k l}\left(\alpha_{i j}\right)=0 .
$$

The actual form of the expression for $\nabla \underline{v}$ depends on the distribution of the variables in space. Among the common staggered grid systems discussed by Arakawa (1972), the A-grid is seldom used in numerical models and the $\mathrm{D}$ - and E-grids are rotations of the $\mathrm{C}$ - and B-grids respectively. Therefore, the detailed derivation of the scheme will be included in this article for the B-and C-grids only. The distribution of variables in the B-grid and the C-grid is showh in Fig. 1 and Fig. 2 respectively.

The $x$ - and $y$-components of a non-divergent wind field are given by

$$
u=-n \frac{\partial \psi}{\partial y}, \quad v=m \frac{\partial \psi}{\partial x}
$$

where $\psi$ is arbitrary. Adopting the following finite difference analogues:

$$
\begin{aligned}
& {\overline{n_{i j}}}^{x y}{\overline{\delta_{y} \psi_{i j}}}^{x} \text { for } n \frac{\partial \psi}{\partial y} \\
& \quad \text { and }{\overline{m_{i j}}}^{x y}{\overline{\delta_{x} \psi_{i j}}}^{y} \text { for } m \frac{\partial \psi}{\partial x}
\end{aligned}
$$

the desired expression for the divergence for the B-grid is

$$
\left.\nabla \underline{v}_{i j}=m_{i j} n_{i j}\left(\overline{\delta_{x}\left(\frac{u_{i j}}{\overline{n_{i j}} x y}\right)}+\overline{\delta_{y}\left(\frac{v_{i j}}{m_{i j}} x y\right.}\right)\right)
$$

which reduces to

$$
\begin{aligned}
& \nabla \underline{v}_{i j}=m_{j}\left({\overline{\delta_{x} u_{i j}}}^{y}+\overline{\left.\delta_{y}\left(\frac{v_{i j}}{\bar{m}_{j}^{y}}\right)^{x}\right)}\right. \text { and } \\
& \nabla \underline{v}_{i j}=m_{j}^{2}\left(\overline{\left(\frac{\delta_{x} u_{i j}}{\bar{m}_{j}^{y}}\right)} y={\overline{\delta_{y}}\left(\frac{v_{i j}}{m_{j}}\right)}^{y}\right)
\end{aligned}
$$

for the latitude-longitude and the Mercator projections respectively. The details of the above computation are listed in Appendix A.

For the C-grid (Fig. 2), the desired expression for the divergence takes the following form:

$$
\nabla \underline{v}_{i j}=m_{i j} n_{i j}\left(\delta_{x}\left(\frac{u_{i j}}{n_{i+1 / 2, j}}\right)+\delta_{y}\left(\frac{v_{i j}}{m_{i, j+1 / 2} y}\right)\right)
$$

which reduces to 

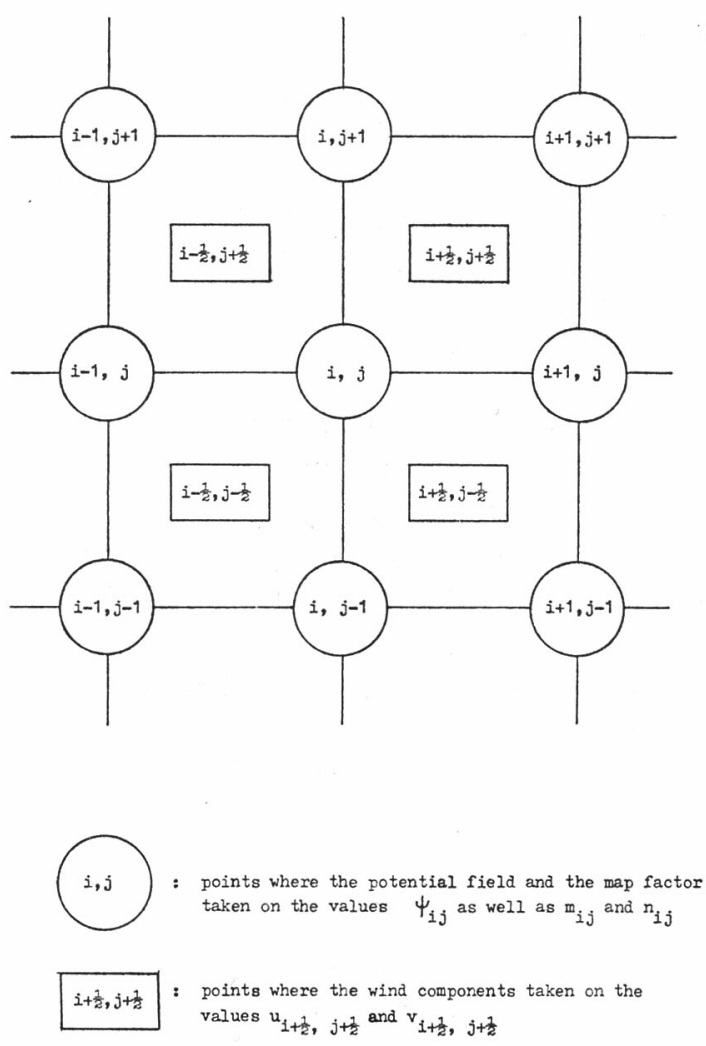

Fig. 1 A portion of the B-grid.

$$
\begin{aligned}
& \nabla \underline{v}_{i j}=m_{j}\left(\delta_{x} u_{i j}+\delta_{y}\left(\frac{v_{i j}}{m_{j+1 / 2}}\right)\right) \text { and } \\
& \nabla \underline{v}_{i j}=m_{j}\left(\delta_{x} u_{i j}+m_{j} \delta_{y}\left(\frac{v_{i j}}{\overline{m_{j+1 / 2}} y}\right)\right)
\end{aligned}
$$

for the latitude-longitude and the Mercator projections respectively. Some details of the above computation are listed in Appendix B.

A comparison among the expressions (2.5) to (2.8) shows that the form of the divergence scheme which will give zero divergence for any non-divergent wind field is dependent on the choice of the finite difference scheme for the non-divergent wind field. An example is quoted in Appendix $C$ which shows that for some choice of the finite difference scheme for the non-divergent wind field, the computed values of the divergence never vanish for any divergence scheme. For other choice of the finite difference scheme for the nondivergent wind field, the divergent scheme
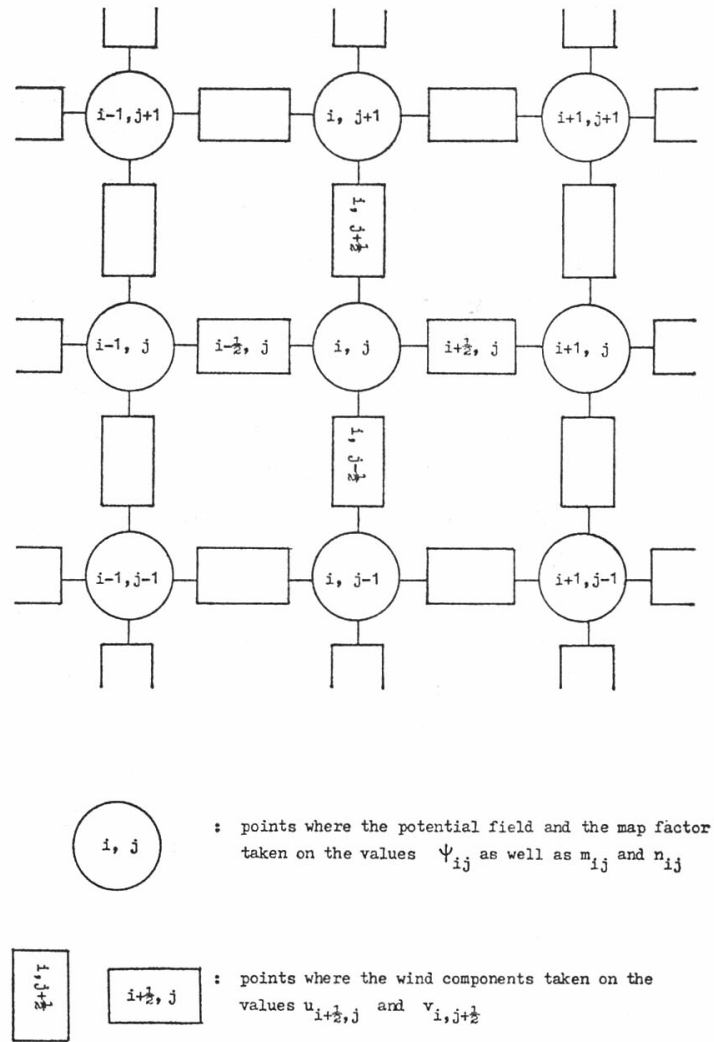

Fig. 2 A portion of the C-grid.

to give zero divergence may become too complicated. An example is quoted in Appendix $\mathrm{D}$.

\section{Results and discussions}

We compare the results of 12- and 24-hour forecasts produced by applying the following scheme

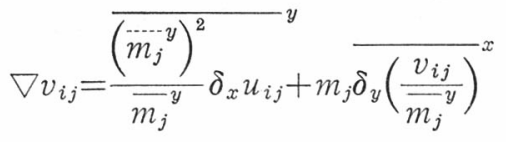

where

$$
\left(m_{j}^{y}\right)^{2}=m_{j-1 / 2} m_{j+1 / 2}
$$

(which will give zero divergence for any nondivergent wind field) to those produced by an ordinary scheme

$$
\nabla \underline{v}_{i j}=m_{j}\left({\overline{\delta_{x} u_{i j}}}^{y}+\overline{\left.\delta_{y}\left(\frac{v_{i j}}{m_{j}}\right)^{x}\right)}\right.
$$

(which may give non-zero divergence for 
some non-divergent wind field).

The numerical model employed (Chan, 1984) is a 3 level limited area primitive equation one in 2.5 degree latitude-longitude B-grid. Hybrid vertical coordinate is used with two $\sigma$-levels beneath and one $p$-level on top. The level $\sigma=0$ corresponds to $p=400$ $\mathrm{hPa}$. A parameterization scheme for cumulus convection similar to that of Kuo (1965) is employed in the model while radiation is neglected. Data are dynamically initialized.

Figs. 3 and 4 show the total mass variation of the 12- and 24-hour forecasts respectively. The larger errors in the values of the divergence when an ordinary scheme is used are reflected in the inaccuracy in the values of the forecast surface pressure. The larger errors associated with the 12-hour forecasts show that quasi-nondivergence is not attained for a 12-hour forecast but is attained once more at the end of a 24-hour integration.

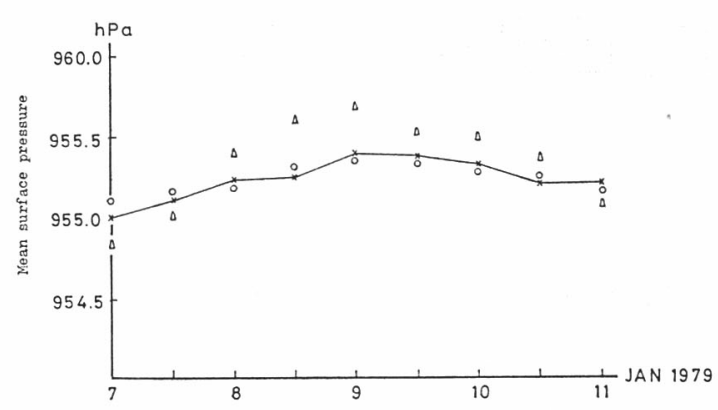

Fig. 3 Variation of total mass (expressed by mean surface pressure). The ' $x$ ' represents the observed values, the ' $O$ ' for applying the designed scheme in a 12 -hour forecasts and the ' $\triangle$ ' for the ordinary scheme.

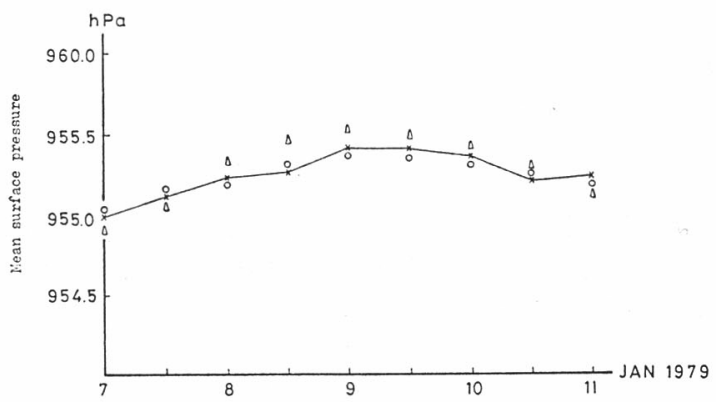

Fig. 4 Same as Fig. 3 except that the comparison is carried out for 24 -hour forecasts.

\section{Summary}

A finite difference scheme is constructed such that it will give zero divergence for any non-divergent wind field. The scheme is applied successfully to a limited area primitive equation model and gives better results than those from the application of an ordinary divergence scheme.

\section{Acknowledgements}

The author would like to express his heartiest gratitude to Dr. K. Y. Chan in the Mathematics Department, University of Hong Kong for the stimulating discussions on the subject and the helpful suggestions.

Thanks are also due to Miss W.Y. Hui for her excellent typing of the paper, and to Miss M. Wai for her beautiful diagrams.

\section{Appendix A}

Let

$$
\begin{aligned}
\nabla \underline{v}_{i j}= & A u_{i+1 / 2, j+1 / 2}+B u_{i+1 / 2, j-1 / 2} \\
& +C u_{i-1 / 2, j+1 / 2}+D u_{i-1 / 2, j-1 / 2} \\
& +A^{\prime} v_{i+1 / 2, j+1 / 2}+B^{\prime} v_{i+1 / 2, j-1 / 2} \\
& +C^{\prime} v_{i-1 / 2, j+1 / 2}+D^{\prime} v_{i-1 / 2, j-1 / 2}
\end{aligned}
$$

Substituting (2.4) for $u$ and $v$ in (A1) and equating the coefficients $C$ of $\psi$ in the resulting expression to zero, we obtain the following system of linear simultaneous equations:

$$
\begin{aligned}
& C_{i+1, j+1}=x_{1}-x_{5}=0 \\
& C_{i+1, j}=x_{1}-x_{2}+x_{5}+x_{6}=0 \\
& C_{i+1, j-1}=x_{2}+x_{6}=0 \\
& C_{i, j+1}=x_{1}+x_{3}+x_{5}-x_{7}=0 \\
& C_{i j}=x_{1}-x_{2}+x_{3}-x_{4}-x_{5}-x_{6}+x_{7}+x_{8}=0 \\
& C_{i, j-1}=x_{2}+x_{4}-x_{6}+x_{8}=0 \\
& C_{i-1, j+1}=x_{3}+x_{7}=0 \\
& C_{i-1, j}=x_{3}-x_{4}-x_{7}-x_{8}=0 \\
& C_{i-1, j-1}=x_{4}-x_{8}=0 .
\end{aligned}
$$

Simplification and rearrangement lead to:

$$
M \underline{x}=\underline{0}
$$

where 


$$
\begin{aligned}
& M=\left(\begin{array}{rrrrrrrr}
1 & 0 & 0 & 0 & -1 & 0 & 0 & 0 \\
0 & 1 & 0 & 0 & 0 & 1 & 0 & 0 \\
0 & 0 & 1 & 0 & 0 & 0 & 1 & 0 \\
0 & 0 & 0 & 1 & 0 & 0 & 0 & -1 \\
0 & 0 & 0 & 0 & 1 & 0 & 0 & 1 \\
0 & 0 & 0 & 0 & 0 & 1 & 0 & -1 \\
0 & 0 & 0 & 0 & 0 & 0 & 1 & 1
\end{array}\right) \\
& \underline{0}=(0,0,0,0,0,0,0)^{T} \\
& \underline{x}=\left(x_{1}, x_{2}, x_{3}, x_{4}, x_{5}, x_{6}, x_{7}, x_{8}\right)^{T}
\end{aligned}
$$

and

$$
\left.\begin{array}{l}
x_{1}={\overline{n_{i+1 / 2, j+1 / 2}}}^{x y} A / \Delta y \\
x_{2}={\overline{n_{i+1 / 2, j-1 / 2}}}^{y} B / \Delta y \\
x_{3}={\overline{n_{i-1 / 2, j+1 / 2}}}^{y} \mathrm{C} / \Delta y \\
x_{4}={\overline{n_{i-1 / 2, j-1 / 2}}}^{x y} D / \Delta y \\
x_{5}={\overline{m_{i+1 / 2, j+1 / 2}}}^{x y} A^{\prime} / \Delta x \\
x_{6}={\overline{m_{i+1 / 2, j-1 / 2}}}^{y} B^{\prime} / \Delta x \\
x_{7}={\overline{m_{i-1 / 2, j+1 / 2}}}^{y} C^{\prime} / \Delta x \\
x_{8}={\overline{m_{i-1 / 2, j-1 / 2}}}^{y} D^{\prime} / \Delta x
\end{array}\right\} .
$$

There are infinitely many permissible solutions to the above system. However, if we require that the expression (A1) gives the corresponding differential expression for divergence in the limit when $\Delta x, \Delta y$ approach zero, one such choice is obtained by putting

$$
\underline{x}=(1,1,-1,-1,1,-1,1,-1)^{T} \alpha
$$

where

$$
\alpha=\frac{m_{i j} n_{i j}}{2 \Delta x \Delta y}
$$

Substituting (A2) and (A3) into (A1), we obtain (2.5). The corresponding expression in latitude-longitude projection is obtained by putting

$$
m_{i j}=m_{j}=\sec ((j-1) y / a), \quad n_{i j}=1 ;
$$

while that in Mercator projection (true at latitude $\varphi_{0}$ ) is obtained by putting

$$
m_{i j}=n_{i j}=m_{j}=\left(\cos ^{2} \varphi_{0}+\left(\frac{j-1}{a}\right)^{2} \Delta y^{2}\right)^{1 / 2} ;
$$

where ' $a$ ' is the radius of the earth.

\section{Appendix B}

Let

$$
\begin{aligned}
\nabla \underline{v}_{i j}= & A u_{i+1 / 2, j}+B u_{i-1 / 2, j} \\
& +C v_{i, j+1 / 2}+D v_{i, j-1 / 2} .
\end{aligned}
$$

Using

$$
\begin{aligned}
& \bar{m}_{i j}^{y} \frac{\bar{\delta}_{x} \psi_{i j}}{x y} \text { for } m \frac{\partial \psi}{\partial x} \\
& \text { and }{\overline{n_{i j}}}^{x}{\overline{\delta_{y} \psi_{i j}}}^{x y} \text { for } n \frac{\partial \psi}{\partial y}
\end{aligned}
$$

similar computation as in Appendix A leads to:

$$
\begin{aligned}
& \left(\begin{array}{cccc}
1 & 0 & 0 & 1 \\
0 & 1 & 0 & -1 \\
0 & 0 & 1 & 1
\end{array}\right)\left(\begin{array}{l}
x_{1} \\
x_{2} \\
x_{3} \\
x_{4}
\end{array}\right)=\left(\begin{array}{l}
0 \\
0 \\
0 \\
0
\end{array}\right) \\
& \left(x_{1}, x_{2}, x_{3}, x_{4}\right)=(1,-1,1,-1) \alpha
\end{aligned}
$$

where

$$
\begin{aligned}
& x_{1}={\overline{n_{i+1 / 2, j}}}^{x} A / \Delta y \\
& x_{2}={\overline{n_{i-1 / 2, j}}}^{x} B / \Delta y \\
& x_{3}={\overline{m_{i, j+1 / 2}}}^{y} C / \Delta x \\
& x_{4}={\overline{m_{i, j-1 / 2}}}^{y} D / \Delta y \\
& \alpha=\frac{m_{i j} n_{i j}}{2 \Delta x \Delta y} .
\end{aligned}
$$

Substituting (B2) and (B3) into (B1) we obtain (2.7). (2.8) then follows similarly.

\section{Appendix C}

Let

$$
\begin{aligned}
\nabla \underline{v}_{i j}= & A u_{i+1 / 2, j}+B u_{i-1 / 2, j} \\
& +C v_{i, j+1 / 2}+D v_{i, j-1 / 2} .
\end{aligned}
$$

Using the following expressions

$$
\begin{aligned}
& {\overline{m_{i j}} \overline{\delta_{x} \psi_{i j}}}^{y} \text { for } m \frac{\partial \psi}{\partial x}
\end{aligned}
$$

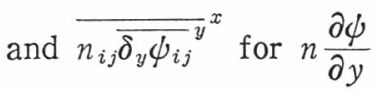

the coefficient for $\phi_{i+1, j+1}, \psi_{i+1, j-1}$ and $\phi_{i-1, j}$ are given by

$$
C_{i+1, j+1}=-\frac{A}{4 \Delta y} n_{i+1, j}+\frac{C}{4 \Delta x} m_{i, j+1}
$$




$$
\begin{aligned}
& C_{i+1, j-1}=\frac{A}{4 \Delta y} n_{i+1, j}+\frac{D}{4 \Delta x} m_{i, j-1} \\
& C_{i-1, j}=-\frac{C}{4 \Delta x} m_{i j}-\frac{D}{4 \Delta x} m_{i j} .
\end{aligned}
$$

Thus

$$
\begin{aligned}
& C_{i+1, j+1}+C_{i+1, j-1} \\
& =\frac{C}{4 \Delta x} m_{i, j+1}+\frac{D}{4 \Delta x} m_{i, j-1} .
\end{aligned}
$$

Unless $m_{i, j+1}=m_{i, j-1},(\mathrm{C} 4)$ and (C5) will be in contradiction when $C_{i+1, j+1}, C_{i+1, j-1}$ and $C_{i-1, j}$ vanish at the same time. Thus, if (C1) is used for the non-divergent wind field, there will be no divergence scheme giving zero divergence for non-divergent wind fields.

\section{Appendix D}

In the B-grid, if we use the following expressions :

$$
\begin{aligned}
& {\overline{\overline{n_{i j}}}}^{y} \delta_{y} \psi_{i j} \\
& \text { and } \overline{{\overline{m_{i j}}}^{x} \delta_{x} \psi_{i j}} \text { for } n \text { for } m \frac{\partial \psi}{\partial x}
\end{aligned}
$$

the coefficient matrix would be in the form

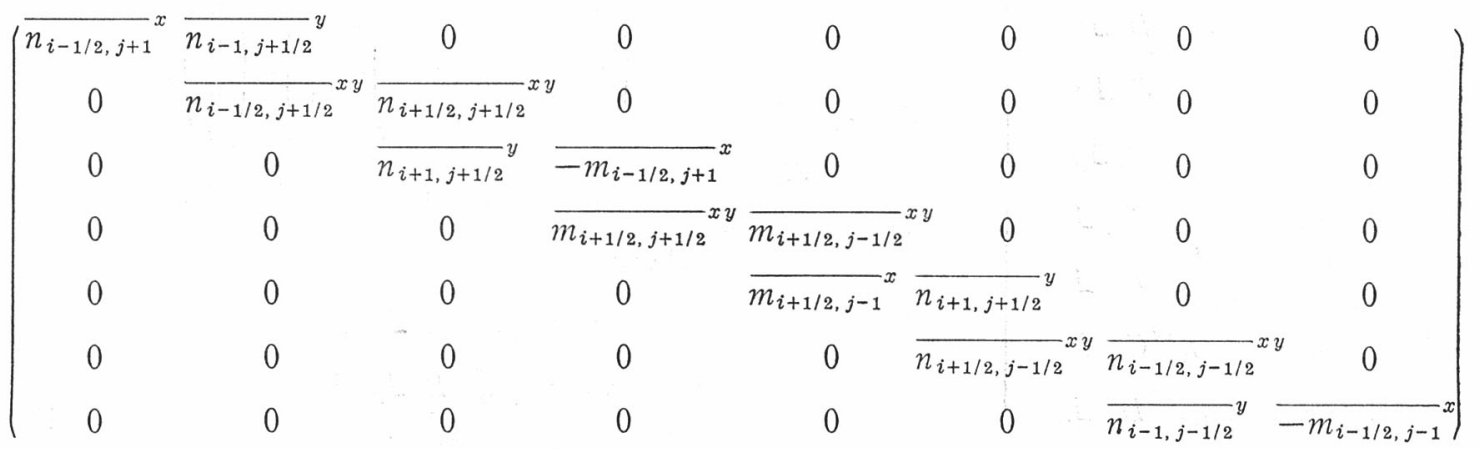

The resulting expression in the divergence is too clumsy to be quoted here. However, the corresponding expressions in latitude-longitude and Mercator projections are relatively simple:

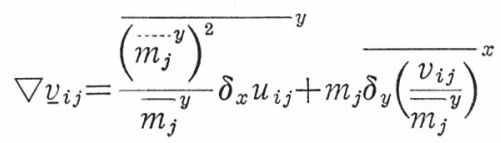

$$
\begin{aligned}
& \nabla \underline{v}_{i j}=m_{j}\left(\frac{1}{{\overline{m_{j+1 / 2}}}^{y}} \frac{{\overline{\left(m_{j}^{y}\right)^{2}}}_{\bar{m}_{j}^{y}}^{y} \delta_{x} u_{i j}+m_{j} \delta_{y}\left(\frac{v_{i j}}{\bar{m}_{j}^{y}}\right)}{x}\right) .
\end{aligned}
$$

\section{References}

Arakawa, A., 1972: Design of the UCLA General Circulation Model. Numerical Simulation of Weather and Climate. Technical Report, No. 7, Dept of Meteorology, UCLA.

Chan, Y.K., 1984: A limited area primitive equation weather prediction model for Hong Kong. M. Phil. Thesis, University of Hong Kong, 1984.

Haltiner, G. J. and R. T. Williams, 1980: Numerical Prediction and Dynamic Meteorology. John Wiley and Sons, New York.

Kuo, H.L., 1965: On formulation and intensification of tropical cyclones through latent heat release by cumulus convection. J. Atmos. Sci., 22, 40-63. 